\title{
Recent developments of Bragg gratings in PMMA and TOPAS polymer optical fibers
}

David J. Webb, Kyriacos Kalli, Karen Carroll, Chi Zhang, Michalis Komodromos, et al.

David J. Webb, Kyriacos Kalli, Karen Carroll, Chi Zhang, Michalis Komodromos, Alex Argyros, Maryanne Large, Grigoriy Emiliyanov, Ole Bang, Eric Kjaer, "Recent developments of Bragg gratings in PMMA and TOPAS polymer optical fibers," Proc. SPIE 6830, Advanced Sensor Systems and Applications III, 683002 (26 November 2007); doi: 10.1117/12.761167

SPIE. Event: Photonics Asia 2007, 2007, Beijing, China 


\title{
Recent developments of Bragg gratings in PMMA and TOPAS polymer optical fibers
}

\author{
David J. Webb ${ }^{\mathrm{a}}$, Kyriacos Kalli ${ }^{\text {** }}$, Karen Carroll ${ }^{\mathrm{a}}$, Chi Zhang ${ }^{\mathrm{a}}$, Michael Komodromos ${ }^{\mathrm{c}}$ \\ Alex Argyros ${ }^{\mathrm{d}}$, Maryanne Large ${ }^{\mathrm{d}}$, Grigoriy Emiliyanove ${ }^{\mathrm{e}}$, Ole Bang ${ }^{\mathrm{e}}$, Erik Kjaer ${ }^{\mathrm{f}}$ \\ ${ }^{a}$ Photonic Research Group, Aston University, Aston Triangle, Birmingham, B4 7ET, UK; \\ banophotonics Research Laboratory, Higher Technical Institute, Nicosia, 2152, Cyprus; \\ ${ }^{\mathrm{c}}$ Frederick Research Centre, 7 Filokyprou, Nicosia, Cyprus ; \\ ${ }^{\mathrm{d} O p t i c a l ~ F i b r e ~ T e c h n o l o g y ~ C e n t r e, ~ U n i v e r s i t y ~ o f ~ S y d n e y, ~ S y d n e y, ~ N S W ~ 2006, ~ A u s t r a l i a ; ~}$ \\ ${ }^{\mathrm{e}}$ Technical University of Denmark, DK-2800 Kgs. Lyngby, Denmark \\ ${ }^{\mathrm{f}}$ Danish Polymer Centre (DTU), Technical University of Denmark, DK-2800 Lyngby, Denmark
}

\begin{abstract}
We report on the temperature response of FBGs recorded in pure PMMA and TOPAS holey fibers. The gratings are fabricated for operational use at near IR wavelengths, using a phase mask and a cw He-Cd laser operating at 325nm. The room temperature grating response is non-linear and characterized by quadratic behavior for temperatures from room temperature to the glass transition temperature, and this permanent change is affected by the thermal history of the gratings. We also report the first FBG inscription in microstructured polymer optical fibers fabricated from TOPAS. This material is fully polymerized and has very low moisture absorption, leading to very good fiber drawing properties. Furthermore, although TOPAS is chemically inert and bio-molecules do not readily bind to its surface, treatment with Antraquinon and subsequent UV activation allows sensing molecules to be deposited in well defined spatial locations. When combined with grating technology this provides considerable potential for label-free bio-sensing.
\end{abstract}

Keywords: Fiber Bragg gratings, polymer optical fiber, temperature response, PMMA, TOPAS, bio-sensing

\section{INTRODUCTION}

Interest in fiber Bragg gratings (FBGs) in polymer optical fiber (POF) has been increasing in recent years, due largely to the expectation that they will open up new applications where FBGs in silica fibers have not been suitable. Gratings have been reported in both step index (SI) [1,2] and microstructured polymer optical fibers (mPOFs) [3]. FBGs in POF are particularly appealing in medical devices as polymers are already routinely put in the human body in many applications. They have already been shown to be more sensitive to both strain and temperature than is the case for silica FBGs [4,5]. The majority of polymer optical fiber (POF) to date is based on polymethylmethacrylate (PMMA). As a potentially low cost sensing solution, it has several advantages compared with its silica counterpart, including higher thermal sensitivity, better inherent material biocompatibility and the ability to survive much higher strain levels. Microstructured optical fibers have a pattern of air holes that extend for the full length of the fiber and the optical properties can be designed by adjusting the relative position, size and shape of the air holes. Importantly, the holes can be used to hold a gas or a biological sample, which can be studied by evanescent-wave sensing. In this family of fibers mPOFs have received particular interest. The lower melting temperature of the polymer, the more flexible hole patterns made possible by drilling the preform and the improved biocompatibility are some of the main advantages over silica based fibers for this application. Monomer residues inside PMMA, which is the usual material for mPOFs, and its aptitude for water absorption often make the drawing process with commercially available PMMA rods problematic. These problems can be reduced by using other polymer materials, such as TOPAS ${ }_{\circledast}$ cyclic olefin copolymers (TOPAS COCs) [6]. TOPAS COCs have no monomers and their moisture absorption is a hundred times lower than PMMA. In addition, they exhibit a low stress-optical constant, which allows for better conditions for the drawing of fibers. Finally, whereas biomolecules bind easily to PMMA, TOPAS is a chemically inert material and direct binding of biomolecules onto its surface is difficult. However, commercially available Antraquinon (AQ) linker molecules can attach to the TOPAS surface when activated by UV light and can subsequently accept sensor layers. Therefore TOPAS has the unique property of allowing 
the use of a UV mask to define local sensor layers to provide selective multi-antibody bio-sensing. With gratings inscribed, such biosensors can even be made label-free.

In this paper, we present recent developments in the temperature characteristics of FBGs in both PMMA and TOPAS $\mathrm{POF}$ and show how these characteristics may be tailored in order to increase the working temperature range of these sensors. While we report on work carried out in $\mathrm{MPOF}$, we have observed similar behavior in SIPOF and therefore the observed behavior should not be viewed as solely applicable to FBGs in mPOF.

\section{PMMA AND TOPAS MPOF GRATING FABRICATION}

In both fiber types FBGs were fabricated in nominally single mode mPOF (SMmPOF) using a cw helium cadmium laser (KIMMON IK5652R-G) operating at $325 \mathrm{~nm}$ and $\sim 30 \mathrm{~mW}$ of laser power. The experimental arrangement is shown in figure 1. The ends of the MPOF were cleaved by using a sharp razor blade and the fiber was mounted into v-grooves, thereby supported along its length in order to prevent sagging. The $\mathrm{v}$-grooves were attached to an $\mathrm{x}, \mathrm{y}, \mathrm{z}$ translation stage. A static laser beam (beam width $=1.8 \mathrm{~mm}$ ) was focused vertically onto the fiber by a $10 \mathrm{~cm}$ cylindrical lens through a phasemask (mask period $=1057.2 \mathrm{~nm}$ ). The grating length was therefore equal to the beam width. The gratings were monitored either in reflection (PMMA) or transmission (TOPAS) during inscription using an L-band broadband light source (BBS) and optical spectrum analyzer (OSA). Light was coupled into the fiber from the L-band BBS and out of the fiber to the OSA by butt coupling to FC/APC connectors.

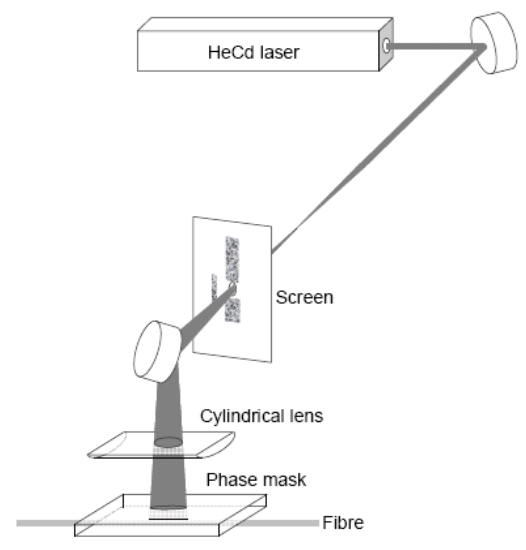

Fig. 1: Experimental arrangement for FBG inscription.

\subsection{PMMA fiber cross section and wavelength spectrum}

A cross section of the PMMA fiber used in this experiment is shown in figure 2(a). The sides of the fiber were flattened in order to investigate whether this geometry would aid alignment of the laser beam relative to the holes in the fiber, however no benefit was observed. The fiber used consisted of pure PMMA and was fabricated using the method described in reference [7]. The resulting grating wavelength was in the order of $1562 \mathrm{~nm}$, which is in a very high loss region of the fiber attenuation spectrum at close to $100 \mathrm{~dB} / \mathrm{m}$. This region was chosen purely for convenience in that most of the equipment available to us operates in this wavelength range. For this reason, short lengths of fiber were used $(\sim 5 \mathrm{~cm})$ and it was not possible to view the gratings in transmission. A typical reflection spectrum of a grating used in this study is shown in figure 2(b). 


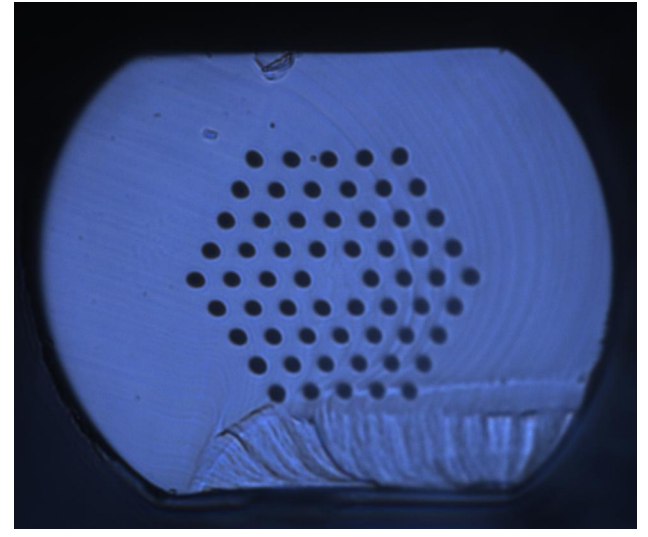

(a)

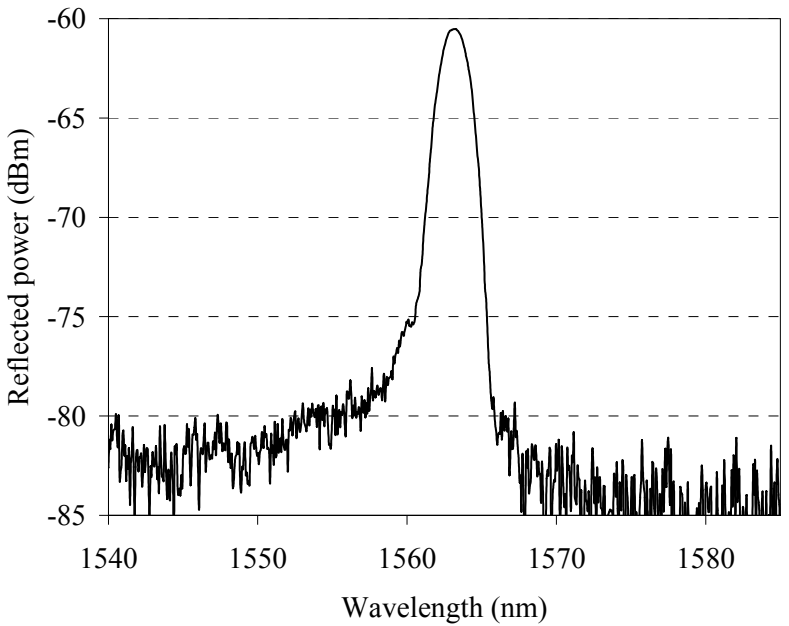

(b)

Fig. 2. (a)Cross section of PMMA SMmPOF used in this experiment, (b) reflection spectrum of a grating fabricated in SMmPOF

\subsection{TOPAS fiber cross section and wavelength spectrum}

The TOPAS fiber was fabricated at COM•DTU at the Technical University of Denmark (DTU), from preforms made at the Danish Polymer Center at DTU. The preform was made in a vacuum oven from TOPAS COC granules of the grade 8007 and had a diameter of $50 \mathrm{~mm}$ and a length of $85 \mathrm{~mm}$. Two rings of $2 \mathrm{~mm}$ holes were then drilled into the preform in a triangular structure and the preform was drawn to fiber at about $140^{\circ} \mathrm{C}$ with no pressure applied to the air holes. The few mode microstructured TOPAS mPOF used in this study had an outside diameter of $400 \mu \mathrm{m}$ and a core diameter of $80 \mu \mathrm{m}$. The core being surrounded by 18 air holes with diameters of $55 \mu \mathrm{m}$, see figure 3(a). Normally it required around 30-40 minutes of exposure to approach a maximum grating reflectivity. The transmission spectra of the gratings were recorded and revealed a Bragg wavelength around $1600 \mathrm{~nm}$, though this varied by several $\mathrm{nm}$ from grating to grating, figure 3(b). From the position of the resonance, we can deduce the effective refractive index of the propagating mode in the TOPAS $\mathrm{mPOF}$ by calculation, which is around 1.513 .

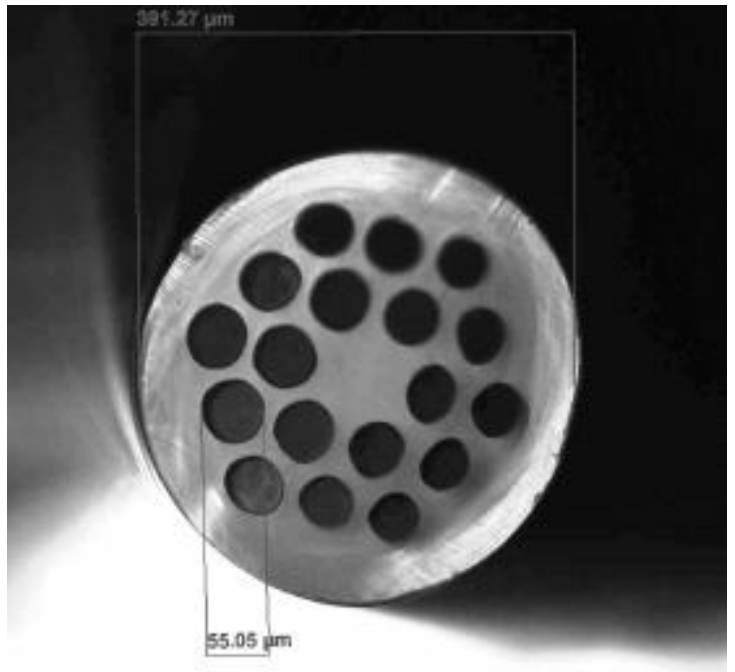

(a)

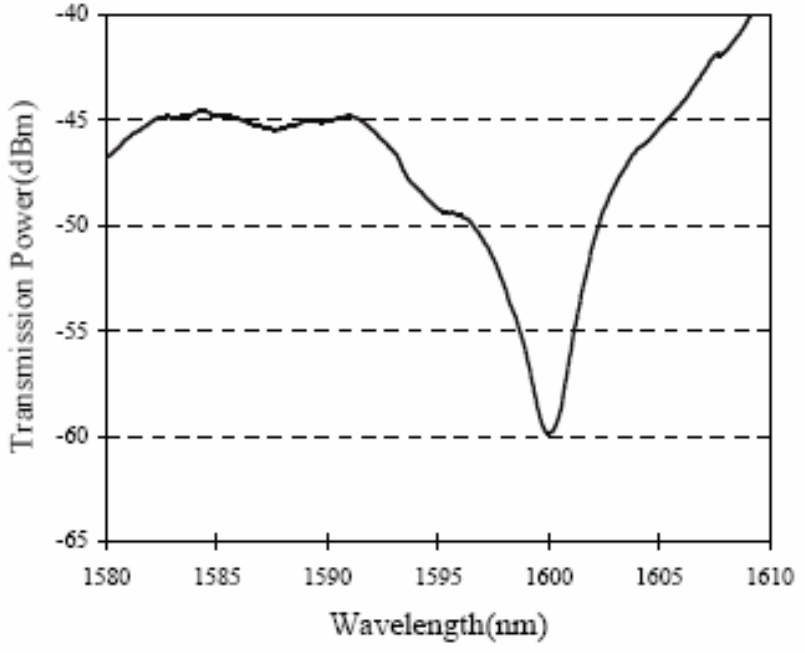

(b)

Fig.3. (a) Optical microscope image of the cross-section of the TOPAS mPOF, (b) transmission profile of fabricated FBG 


\section{THERMAL RESPONSE OF PMMA AND TOPAS MPOF GRATINGS}

The thermal response of the gratings was investigated using the heating rig shown in figure 4 [8]. Two Melles Griot $\mathrm{v}$ groove blocks were fastened on a translation stage leaving a $1 \mathrm{~cm}$ long air gap between them. The mPOF with FBG was placed in the v-grooves along with a temperature calibrated FBG in silica fiber, which was used to measure the temperature of the heating zone, making sure the two FBGs were closely spaced in the air gap. A high power resistor $(25 \mathrm{~W}, 100 \mathrm{~W}$, ) was placed over the FBGs to heat the devices and the whole heating zone was insulated by polystyrene pieces. The heating process was monitored by the same arrangement as the grating inscription. The FBG region was generally heated to several temperatures by tuning the voltage value across the resistor. At each new settings, the voltage was held constant for 10 minutes to ensure temperature stabilize before the value of the shift in the peak of the grating spectrum was recorded from the OSA.

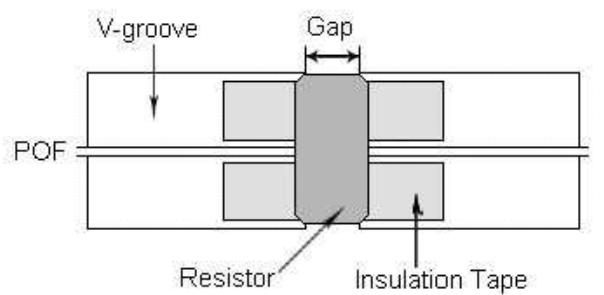

(a)

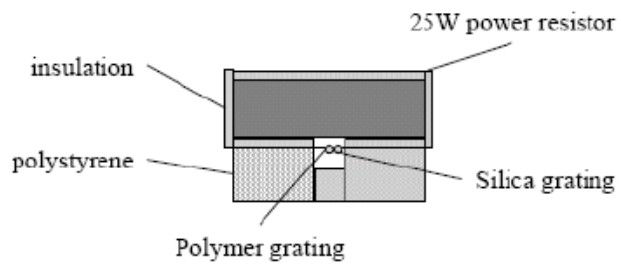

(b)

Fig. 4. (a) Top view of the heating rig, (b) cross-section view of the heating rig.

\subsection{Thermal properties of FBGs in PMMA}

The first experiment consisted of cycling a single grating, made in non-annealed fiber, to different temperatures, figure 5. The grating was first taken to a maximum of $77^{\circ} \mathrm{C}$ with each incremental temperature being held for 10 minutes. The grating was then allowed to cool back to room temperature. In the second cycle, the grating was heated to $86^{\circ} \mathrm{C}$, cooled again, then in the final cycle the maximum temperature was $92^{\circ} \mathrm{C}$. After each cycle, a permanent shift in the wavelength of the grating was observed; after the first cycle this was $-8.4 \mathrm{~nm}$ and after the second this was a further $-10.1 \mathrm{~nm}$. The temperature response of each cycle exhibited a quasi-linear region at low temperatures. ] This quasi-linear region extends to higher temperatures with each progressive cycle due to the annealing which took place during the previous cycle. The sensitivity of the grating in each of these regions is shown on the graph in figure 5, and ranges from $-77 \mathrm{pm} /{ }^{\circ} \mathrm{C}$ to $95 \mathrm{pm} /{ }^{\circ} \mathrm{C}$. Although we have referred to a quasi-linear region, the response in this region was actually found to be nonlinear. 


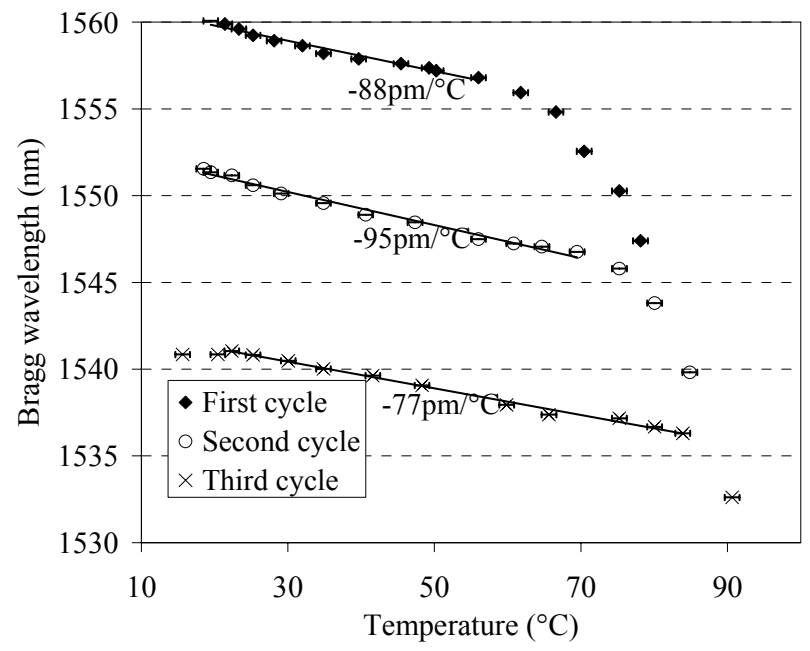

Fig. 5. Bragg wavelength shift with temperature for three consecutive heating cycles. $\bullet$ First cycle, $\bigcirc$ second cycle, third cycle.

The deviation from a linear response for each cycle is presented in figure 6, showing that the response becomes progressively more linear with each cycle. The reason for this nonlinearity is unknown and requires more detailed investigation. Similar nonlinearities have been reported for silica [9] where there is much more information available on the variation of refractive index with temperature.

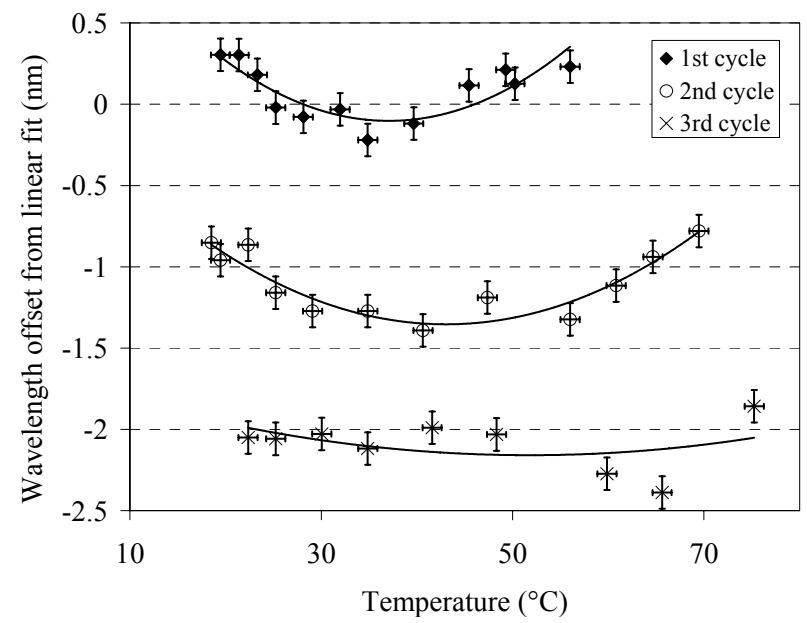

Fig. 6: Deviation from linear response for each cycle. The results are offset by $-1 \mathrm{~nm}$ for each cycle for clarity.

As annealing the fiber after the gratings have been written has some detrimental effect on the strength of the grating peak, a grating was written in fiber which had been heated (or preannealed) for 7 hours at $80^{\circ} \mathrm{C}$. This grating was then heated using the method described previously and the results compared with those for fiber which had not been annealed prior to grating inscription. The results are shown in figure 7. The response of the grating which was made in the preannealed fiber is linear to above $80^{\circ} \mathrm{C}$. 


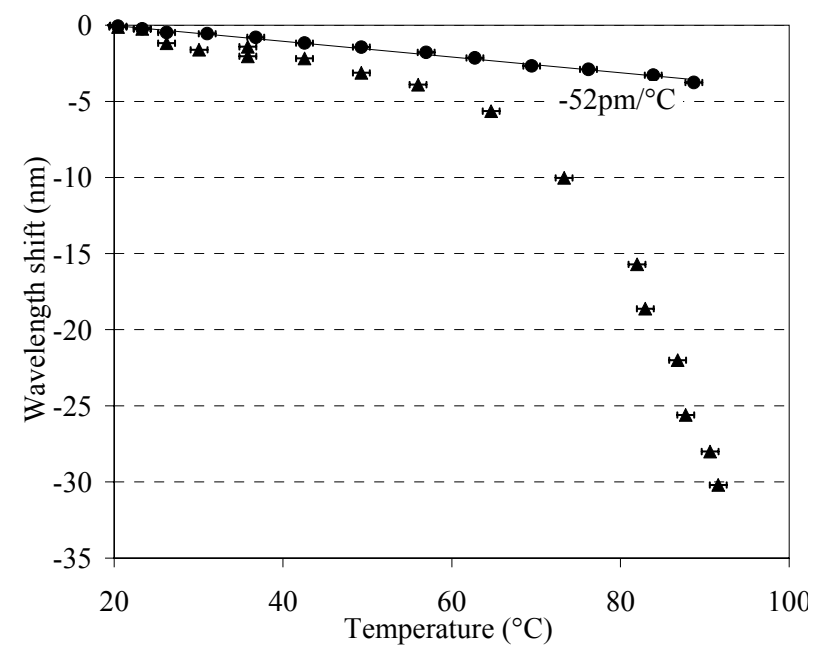

Fig. 7. Comparision between the thermal response of gratings in preannealed fiber and non-annealed fiber.

In order to understand the annealing process, samples of fiber were heated at temperatures ranging from $60^{\circ} \mathrm{C}$ to $100^{\circ} \mathrm{C}$ for 7 hours prior to grating inscription using the method described earlier in this paper. The length and diameter of the fiber was monitored during heating. Three different samples of fiber were measured at each temperature and the mean length change calculated. The results for the change in length with time for different temperatures are shown in figure 8. At temperatures above $80^{\circ} \mathrm{C}$, the length decrease increases significantly. Furthermore, the results also show that even after 7 hours, the fiber is still shrinking.

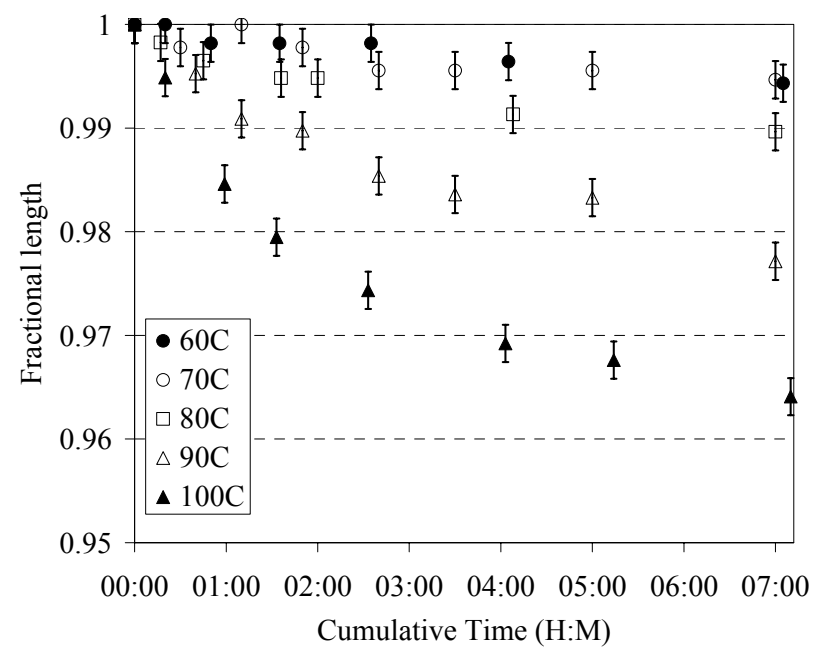

Fig. 8. Change in fiber length with time for different annealing temperatures

The length and diameter results were combined in order to investigate whether there was likely to be any change in density of the fiber during shrinking. The results are shown in figure 9 and indicate that there is no measurable change in density, and thus refractive index, of the fiber during the annealing process. This result suggests that the most significant effect causing a permanent change in the grating wavelength during heating is the change in grating period due to shrinkage of the fiber, rather than any significant change in refractive index. 


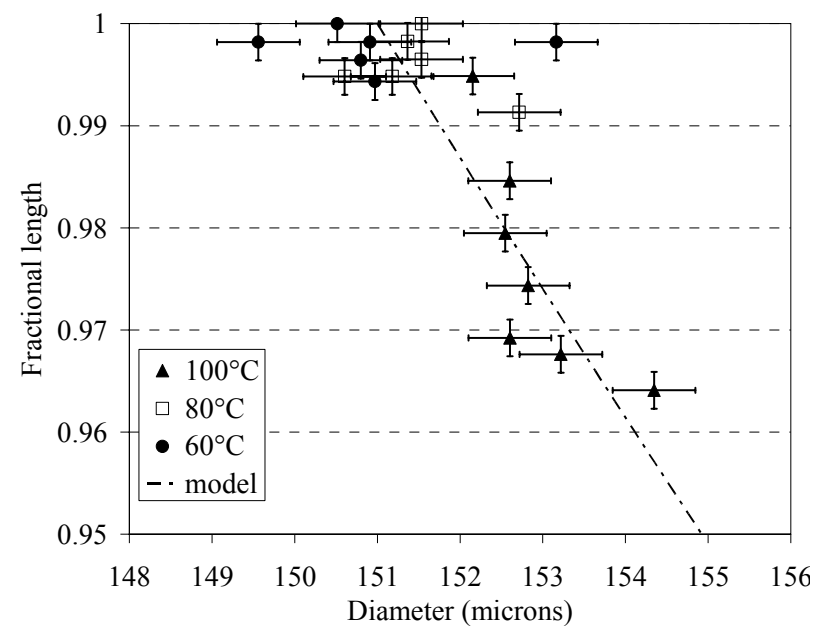

Fig. 9. Fractional length vs diameter for fibers heated at different temperatures. The model line shows the relationship between length and diameter for zero change in density.

\subsection{Thermal properties of FBGs in TOPAS}

Similar measurements were carried out using the heating rig shown in figure 4 for the FBGs inscribed in TOPAS fiber. Typical results are shown in figure 10, which reveals a positive temperature coefficient of $0.81 \mathrm{~nm} /{ }^{\circ} \mathrm{C}-$ to the best of the authors' knowledge, this value is the highest temperature sensitivity of any FBG based device yet reported. However, we also note that this appears to be dependent on the fiber structure, with the highest sensitivity recorded for two-ring TOPAS fiber, whereas three-ring TOPAS $\mathrm{mPOF}$ has a measured wavelength shift of $>250 \mathrm{pm} /$ degree Celsius. Note that in PMMA, FBGs display a negative wavelength shift with rising temperature. [4] This can be explained by the equation describing the Bragg wavelength shift with temperature [10],

$$
\Delta \lambda_{B}=\lambda_{B}(n \alpha+\beta) \Delta T
$$

where $\Delta \lambda_{\mathrm{B}}$ is the Bragg wavelength shift, $\Delta \mathrm{T}$ is the temperature change, $\lambda_{\mathrm{B}}$ is the period of Bragg grating, $\mathrm{n}$ is the average effective core mode index in the grating area, $\alpha$ is the thermal expansion coefficient and $\beta$ is the thermo-optic coefficient. The thermal expansion and thermo-optic properties of the material decides the different Bragg wavelength shift directions.

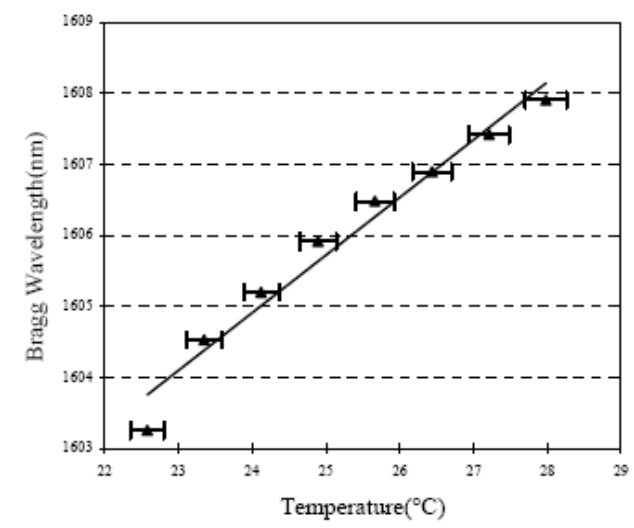

Fig. 10. Bragg wavelength shift with temperature of grating in TOPAS mPOF. 


\subsection{Discussion of results}

For PMMA the results presented show a quasi-linear decrease of Bragg wavelength with increasing temperature below a transition temperature. This transition temperature is dependant on the thermal history of the fiber, as well as the fiber draw parameters [11] and UV exposure [12]. The exact mechanism associated with this transition is not known, though it can be largely explained by the fiber shrinking. This shrinkage is caused by the release of the strain stored in the fiber during the pulling process. We have found that the temperature range of the transition region for non-annealed fiber coincides with that identified by Law as resulting in the best cleaves using a hot fiber/hot blade technique [13]. This shrinkage results in a permanent wavelength shift, which is dependant both on the maximum temperature to which the grating has been cycled, and the duration of heating. Thermal shrinkage tests on lengths of fiber without gratings indicate that there is no measurable change in density as a result of the shrinkage. Annealing of the fiber prior to grating inscription shifts this transition to a higher temperature dependant on the annealing temperature.

Furthermore, the reported thermo-optic coefficient for PMMA is around $-1 \times 10^{-4} \mathrm{~K}^{-1}$ at room temperature but varies considerably with temperature, increasing in magnitude by about $40 \%$ over $40 \mathrm{~K}$ [14]. Quoted values for $\alpha$ vary between $0.7 \times 10^{-4} \mathrm{~K}^{-1}$ and $2.2 \times 10^{-4} \mathrm{~K}^{-1}[4,15-17]$. By choosing different values above, we can get either a negative or a positive Bragg wavelength shift with temperature. However, to the authors' knowledge, no positive Bragg wavelength shift has yet been observed with PMMA. For the TOPAS material used in this study, the value of $\beta\left(-10^{-4} \mathrm{~K}^{-1}\right)$ [18] is also negative, but it is slightly smaller than $n \alpha\left(1.53 \times 0.7 \times 10^{-4} \mathrm{~K}^{-1}\right)[18]$ leading to a calculated positive Bragg wavelength shift of $11 \mathrm{pm} /{ }^{\circ} \mathrm{C}$, which is much smaller than the experimental result. It should be noted though that the values for the thermal expansion coefficient and thermo/optic coefficient obtained from reference [18] are only quoted to one significant figure, which leads to very large errors when the data are combined in equation 1 to calculate the thermal sensitivity. The data also refer to bulk material and the properties may well have been changed during the fiber drawing process. The discrepancy between the calculated and measured temperature sensitivities will be the subject of further work. In our experiments, the maximum temperature we could reach is $28{ }^{\circ} \mathrm{C}$, before the grating visibility became zero. This is much lower than we managed to reach in PMMA based mPOF, where gratings can survive up to $100{ }^{\circ} \mathrm{C}$ [8]; however the problem is likely to be an alignment issue as the grating reappears when the temperature is reduced back to ambient. This issue is currently under investigation.

\section{CONCLUSIONS}

We have presented the wavelength to temperature response for FBGs in both PMMA and TOPAS microstructured polymer optical fibers. In PMMA, we have observed a permanent operational wavelength shift of the grating that occurs once the fiber is taken above a certain threshold temperature. We have also shown that it is possible to shift this threshold temperature to higher temperatures by annealing the POF prior to grating inscription. A quasi-linear linear wavelength response to temperature is demonstrated after successive heating cycles that can be accounted for by fiber shrinkage. We have also successfully inscribed Bragg grating in TOPAS microstructured polymer optical fiber, observing a large positive Bragg wavelength shift with temperature in transmission. This material is fully polymerized and has very low moisture absorption, when combined with grating technology this provides considerable potential for label-free biosensing.

\section{ACKNOWLEDGEMENT}

The authors acknowledge funding for this work from the UK Engineering and Physical Sciences Research Council and the Eureka project "POLYFILTRO".

*kkalli@cytanet.com.cy; phone 35722406537; fax 35722406545 


\section{REFERENCES}

${ }^{1}$ Z.Xiong, G.D.Peng, B.Wu, and P.L.Chu, "Highly tunable Bragg gratings in single-mode polymer optical fibers, ” IEEE Photon. Technol. Lett. 11, 352-354 (1999).

2 J.M.Yu, X.M.Tao, and H.Y.Tam, "Trans-4-stilbenemethanol-doped photosensitive polymer fibers and gratings, " Opt. Lett. 29, 156-158 (2004).

${ }^{3}$ H.Dobb, D.J.Webb, K.Kalli, A.Argyros, M.C.J.Large, and M.A.van Eijkelenborg, "Continuous wave ultraviolet lightinduced fiber bragg gratings in few- and single-mode microstructured polymer optical fibers, " Opt. Lett. 30, 3296-3298 (2005).

${ }^{4}$ H.Y.Liu, G.D.Peng, and P.L.Chu, "Thermal tuning of polymer optical fiber bragg gratings, ” IEEE Photon. Technol. Lett. 13, 824 (2001).

${ }^{5}$ H.B.Liu, H.Y.Liu, G.D.Peng, and P.L.Chu, "Strain and temperature sensor using a combination of polymer and silica fibre bragg gratings," Opt. Commun. 219, 139-142 (2003)

${ }^{6}$ G. Emiliyanov, J.B. Jensen, O. Bang, A. Bjarklev, P.E. Hoiby, L.H. Pedersen, E. Kjaer, and L. Lindvold, "Localized biosensing with Topas microstructured polymer optical fiber", Optics Letters 32, 460-462 (2007); Erratum ibid, 1059 (2007).

${ }^{7}$ G.Barton, M.A.van Eijkelenborg, G.Henry, M.C.J.Large, and J.Zagari, "Fabrication of microstructured polymer optical fibers, ” Opt. Fiber Technol. 10, 325-335 (2004).

${ }^{8}$ Karen E. Carroll, Chi Zhang, David J. Webb, Kyriacos Kalli, Alexander Argyros, and Maryanne C. Large, "Thermal response of Bragg gratings in PMMA microstructured optical fibers," Optics Express, 15, 14, 8844-8850 (2007).

${ }^{9}$ G.M.H.Flockhart, R.R.J.Maier, J.S.Barton, W.N.MacPherson, J.D.C.Jones, K.E.Chisholm, L.Zhang, I.Bennion, I.Read, and P.D.Foote, "Quadratic behavior of fiber Bragg grating temperature coefficients," Appl. Opt. 43, 2744-2751 (2004)

${ }^{10}$ A. Othonos and K. Kalli Fiber Bragg gratings: fundamentals and applications in telecommunications and sensing (Artech House, 1999).

${ }_{11}$ T.Ishigure, M.Hirai, M.Sato, and Y.Koike, "Graded-index plastic optical fiber with high mechanical properties enabling easy network installations. I, ” J. Appl. Polym. Sci. 91, 404-409 (2004).

12 E.E.Shafee, "Effect of photodegradation on the [beta]-relaxation in poly(methylmethacrylate), " Polym. Degrad. Stabil. 53, 57-61 (1996).

13 S.H.Law, M.A.van Eijkelenborg, G.W.Barton, C.Yan, R.Lwin, and J.Gan, "Cleaved end-face quality of microstructured polymer optical fibres," Opt. Commun. 265, 513-520 (2006).

${ }^{14}$ J. M. Cariou, J. Dugas, L. Martin, and P. Michel, "Refractive-index variations with temperature of PMMA and polycarbonate,"Appl. Opt. 25, 334 (1986).

${ }^{15}$ A. Fender, M. Silva-López, W. N. MacPherson, J. S. Barton, J. D. C. Jones, D. Zhao, H. Dobb, D. J. Webb, L. Zhang, and I. Bennion, "Strain and temperature sensitivity of a single-mode polymer optical fiber," Opt. Lett. 30, 3129-3131 (2005).

${ }^{16}$ Z.Y. Zhang, P. Zhao, P. Lin, F.G. Sun, "Thermo-optic coefficients of polymers for optical waveguide applications," Polymer, 47,14, 4893-4896 (2006).

${ }^{17}$ S. H. Goods, R. M. Watson, and M. Yi, "Thermal Expansion and Hydration Behavior of PMMA Molding Materials for LIGA Applications, "SAND REPORT, SAND2003-8000, Sandia National Laboratories, 2003.

${ }^{18}$ Topas ${ }^{\circledR}$ cyclic olefin copolymer Product Specification Document (Ticona Inc.). 